\title{
Intracellular Activity of Antibiotics in a Model of Human THP-1 Macrophages Infected by a Staphylococcus aureus Small-Colony Variant Strain Isolated from a Cystic Fibrosis Patient: Study of Antibiotic Combinations ${ }^{\nabla} \dagger$
}

\author{
Hoang Anh Nguyen, ${ }^{1}$ Olivier Denis, ${ }^{2}$ Anne Vergison, ${ }^{3}$ Paul M. Tulkens, ${ }^{1}$ \\ Marc J. Struelens, ${ }^{2}$ and Françoise Van Bambeke ${ }^{1 *}$ \\ Université Catholique de Louvain, Louvain Drug Research Institute, Unité de Pharmacologie Cellulaire et Moléculaire, ${ }^{1}$ and \\ Hôpital Erasme, Department of Microbiology, Laboratoire de Référence MRSA-Staphylocoques, ${ }^{2}$ and Hôpital des Enfants \\ Reine Fabiola, Département de Maladies Infectieuses Pédiatriques, ${ }^{3}$ Université Libre de Bruxelles, Brussels, Belgium
}

Received 26 August 2008/Returned for modification 10 December 2008/Accepted 17 January 2009

\begin{abstract}
In a companion paper (H. A. Nguyen et al., Antimicrob. Agents Chemother. 53:1434-1442, 2009), we showed that vancomycin, oxacillin, fusidic acid, clindamycin, linezolid, and daptomycin are poorly active against the intracellular form of a thymidine-dependent small-colony variant (SCV) strain isolated from a cystic fibrosis patient and that the activity of quinupristin-dalfopristin, moxifloxacin, rifampin, and oritavancin remains limited (2- to 3-log CFU reduction) compared to their extracellular activity. Antibiotic combination is a well-known strategy to improve antibacterial activity, which was examined here against an intracellular SCV strain using combinations with either rifampin or oritavancin. Time-kill curve analysis using either concentrations that caused a static effect for each antibiotic individually or concentrations corresponding to the maximum concentration in human serum showed largely divergent effects that were favorable when antibiotics were combined with rifampin at low concentrations only and with oritavancin at both low and high concentrations. The nature of the interaction between rifampin, oritavancin, and moxifloxacin was further examined using the fractional maximal effect method, which allows categorization of the effects of combinations when dose-effect relationships are not linear. Rifampin and oritavancin were synergistic at all concentration ratios investigated. Oritavancin and moxifloxacin were also synergistic but at high oritavancin concentrations only. Rifampin and moxifloxacin were additive. This approach may help in better assessing and improving the activity of antibiotics against intracellular SCV strains.
\end{abstract}

Small-colony variant (SCV) strains of Staphylococcus aureus are notoriously difficult to eradicate with most commonly used antistaphylococcal agents (30). Failures favor both selection and acquisition of antibiotic resistance because SCV strains are hypermutators (8). In the companion paper (21), we compared the intracellular activity of a series of 13 antibiotics against a methicillin-susceptible thymidinedependent SCV strain isolated from a cystic fibrosis (CF) patient. We showed that most of these antibiotics are poorly active, with only moxifloxacin, rifampin, and oritavancin able to decrease the intracellular inoculum to less than $1 \%$ of the initial value.

Antibiotic combination is a well-known strategy both to prevent the emergence of resistant organisms and to increase activity against extracellular organisms thanks to additive, and even sometimes synergistic, effects between coadministered drugs $(12,23)$. Available data suggest that combining antibiotics can improve intracellular activity against both normal and SCV phenotypes of $S$. aureus strains (2-4). These studies, how-

\footnotetext{
* Corresponding author. Mailing address: Unité de Pharmacologie Cellulaire et Moléculaire, Université Catholique de Louvain, UCL73.70 avenue Mounier 73, 1200 Brussels, Belgium. Phone: 32276473 78. Fax: 32276473 73. E-mail: francoise.vanbambeke@uclouvain.be.

$\dagger$ Supplemental material for this article may be found at http:/aac .asm.org/.

${ }^{\nabla}$ Published ahead of print on 2 February 2009.
}

ever, used only a limited number of antibiotics and fixed concentrations, making it difficult to appreciate the exact nature of interactions between combined drugs. We therefore undertook to reexamine this issue by including a series of commonly used antibiotics. These were combined with either rifampin, systematically used in most combinations (24), or with oritavancin, a lipoglycopeptide with marked bactericidal activity toward $S$. aureus $(22,26)$; oritavancin is currently in late clinical development (9), and it was one of the most effective drugs in our model (21). In the present study, antibiotic combinations were first tested at two fixed concentrations selected to provide meaningful microbiological and pharmacodynamic information. The most promising combinations were then tested using the fractional maximal effect (FME) approach (10, 16), a method that allows analysis of the effects of combinations when dose-effect relationships are not linear (21) and that uses calculated rather than arbitrarily chosen concentration ratios. The study demonstrates that the combination of rifampin and oritavancin allows nearly complete eradication of an intracellular SCV strain, and it calls for a systematic assessment of antibiotic combinations in difficult-to-treat infections caused by these variants of $S$. aureus.

\section{MATERIALS AND METHODS}

Bacterial strain, intracellular infection, antibiotics, determination of intracellular activity, and statistical analyses. All methods, except those specifically related to the antibiotic combinations described in this paper, were as described 
in the companion paper (21). In brief, experiments were conducted with a stable, thymidine-dependent, fully susceptible SCV strain of methicillin-susceptible $S$. aureus isolated from a chronically infected CF patient and used to infect THP-1 macrophages. Intracellular infection was performed as described previously (5), except that the number of CFU was determined after a 48-h incubation of cell lysate on brain heart infusion agar. Intracellular activity $(E)$ was defined as the decrease in the number of CFU recorded at a given time after phagocytosis and addition of antibiotic(s) at specified concentration(s) in comparison with the postphagocytosis inoculum and is expressed in $\log _{10}$ units. A contribution from extracellular bacteria liberated from lysed macrophages in these counts can be ruled out for the following reasons: (i) extracellular bacteria were eliminated by a washing step at the end of the incubation; (ii) cultures were maintained with antibiotics at concentrations exceeding their MICs, preventing extracellular growth; and (iii) no major loss of macrophage viability was observed as assessed by the measure of the release of lactate dehydrogenase and by the maintenance or even an increase in cell protein (suggestive of cell stasis or growth over the incubation period).

These $E$ values are negative because they pertain to decreases in bacterial counts; a greater activity is therefore associated with a more negative $E$ value. Since this is rather counterintuitive, we will use the absolute values of $E$ when comparing activities throughout this paper. (Note however that the tables in both this paper and the companion paper show actual values and not absolute values.)

Assessment of the effect of antibiotic combinations. Two successive approaches were followed. In the first instance, antibiotics were combined as follows: (i) at their respective static concentrations (i.e., the extracellular concentration causing no apparent change in the intracellular CFU count compared to the postphagocytosis inoculum $\left[C_{s}\right]$, as obtained from previous dose dependence experiments with each antibiotic [see Table 1 in this paper for values and Table 2 in reference 21 for pharmacological descriptions of concentration-effect relationships]) and (ii) at an extracellular concentration corresponding to the maximal serum concentration (total drug) observed in patients after administration of conventional doses of the corresponding antibiotic to humans ( $\left[C_{\max }\right]$ see Table 2 in this paper for individual values and Table 1 in reference 21 for references). To check that the increases in activity seen when antibiotics are combined at their $C_{\max }$ values were not due to a carryover effect upon plating of cell lysates containing high intracellular concentrations of antibiotics, we compared bacterial counts from cultures exposed to cell lysates incubated for $24 \mathrm{~h}$ with antibiotics at their $C_{\max }$ values; samples were then either left untreated or treated with an equal volume of $25 \mathrm{mg} / \mathrm{ml}$ activated charcoal suspension (6). No difference was seen between samples treated with charcoal or left untreated or between samples exposed to cell lysates challenged by antibiotics or to unchallenged lysates, ruling out that any carryover effect could take place under our experimental conditions.

In a second instance, selected combinations were examined in detail using the FME approach $(10,16)$. In the present study, all measurements were made after a 24-h incubation, and the decrease in the intracellular bacterial counts $(E)$ was used as the end point. The $E_{\max }$, i.e., the maximum decrease in the inoculum, was extrapolated from Hill's function for an infinitely large antibiotic concentration after a 24-h incubation (in log decrease CFU at $24 \mathrm{~h}$; values are 1.72 for rifampin, 1.32 for moxifloxacin, and 0.43 and 3.13 for oritavancin, where the two successive values reflect a bimodal effect [see Table 2 in reference 21]); the $E_{\max }$ was taken as the activity level corresponding to an FME of 1 (all $E_{\max }$ values used in the present study correspond to bacterial counts that are above the limit of detection). The concentrations of each antibiotic expected to yield an FME of $0.1,0.3,0.5,0.7$, or $0.9\left(C_{\mathrm{xp}}\right)$ when given alone were then calculated using equation 1 :

$$
C_{\text {xp }}=\frac{\mathrm{FME} \times \mathrm{EC}_{50}}{1-\mathrm{FME}}
$$

where $\mathrm{EC}_{50}$ is the extracellular antibiotic concentration (mg/liter) causing a reduction of the inoculum to halfway between the number of CFU extrapolated for an infinitely low antibiotic concentration $\left(E_{\min }\right)$ and $E_{\max }$ in $24 \mathrm{~h}\left(\mathrm{EC}_{50}\right.$ values, in $\mathrm{mg} / \mathrm{liter}$, are 0.02 for rifampin, 0.3 for moxifloxacin, and 0.6 and 19 for the two successive parts of the regressive function fitted to the oritavancin data [converted in $\mathrm{mg} /$ liter from $\mathrm{EC}_{50}$ values expressed in multiples of the $\mathrm{MIC}$ as shown in Table 1 of the companion paper]). The validity of the model was tested by running experiments at each of these concentrations and comparing the observed values of FME $\left(\mathrm{FME}_{\mathrm{obs}}\right.$; defined as $\left.E / E_{\max }\right)$ to the expected FME $\left(\mathrm{FME}_{\mathrm{xp}}\right)$. To assess the effect of combining two antibiotics, antibiotic $A$ was added at five increasing concentrations $\left(C_{\mathrm{xp} A}\right)$ expected to yield FMEs of $0.1,0.3$, $0.5,0.7$ and 0.9 , whereas antibiotic $B$ was added at concentrations $\left(C_{\mathrm{xp} B}\right)$ expected to provide the complement $(0.9,0.7,0.5,0.3$, and 0.1$)$, so that the expected total $\left(\mathrm{FME}_{\mathrm{xp}(A+B)}\right)$ should always be equal to 1 . The level of intracellular activity expected for an additive effect ( $E_{\mathrm{xp}}$ additive $)$ of antibiotics $A$ and $B$ was then calculated using Katzper's formula (10), shown as equation 2:

$$
E_{\mathrm{xp} \text { additive }}=\frac{\left(E_{\mathrm{max} A} \times C_{\mathrm{xp} A} / \mathrm{EC}_{50 A}\right)+\left(E_{\mathrm{max} B} \times C_{\mathrm{xp} B} / \mathrm{EC}_{50 B}\right)}{\left(1+C_{\mathrm{xp} A} / \mathrm{EC}_{50 \mathrm{~A}}+\mathrm{C}_{\mathrm{xp} B} / \mathrm{EC}_{50 B}\right)}
$$

and compared to the measured level of activity $(E)$ to calculate the actual FME $\left(\mathrm{FME}_{\mathrm{obs}(A+B)}\right)$ of the combination using equation 3:

$$
\mathrm{FME}_{\mathrm{obs}(A+B)}=\frac{E}{E_{\text {xp additive }}}
$$

Values of $\mathrm{FME}_{\mathrm{obs}(A+B)}$ were then plotted as a function of the concentration ratios of the two drugs expected to give an FME of 1 (from 0.1/0.9 to 0.9/0.1), together with the values of the $\mathrm{FME}_{\mathrm{obs}}$ of each antibiotic alone using its actual concentration. In these graphs, a synergistic effect will yield an ordinate value of $>1$, an additive effect yields a value of 1 , an indifferent effect yields a value $<1$ but higher than the values of the of $\mathrm{FME}_{\mathrm{obs}}$ of the corresponding antibiotics alone, and an antagonistic effect has a value of $<1$ and below the $\mathrm{FME}_{\mathrm{obs}}$ of the corresponding antibiotic alone (10).

\section{RESULTS}

Combinations at fixed concentrations. In a first approach, antibiotics were combined at two fixed, arbitrarily chosen concentrations, namely, their static concentrations $\left(C_{s}\right)$ or at a concentration corresponding to their respective $C_{\max }$ (total drug). The results are first illustrated in Fig. 1 for studies examining the changes in the intracellular bacterial counts over time for rifampin and oritavancin taken as typical examples of a combination yielding a beneficial effect compared to what was obtained with each of these antibiotics alone. Based on the experiments made with the static concentrations of each drug (Fig. 1), the benefit of the combination clearly appears as a decrease in the number of CFU of about $1.2 \operatorname{logs}$ at 5 or $24 \mathrm{~h}$ compared to no change for each antibiotic used alone (the model did not allow us to examine longer exposure times because of an overwhelming growth of extracellular bacteria after $24 \mathrm{~h}$ as a result of the low antibiotic concentrations used). The benefit of the combination was also evident when the two antibiotics were used at extracellular concentrations corresponding to their respective $C_{\max }$ values (Fig. 1). Of note is that the combination of rifampin and oritavancin decreased the 72-h CFU count to the limit of detection (4.5 logs), an effect that, so far, has never been seen in our model for an antibiotic given alone, regardless of its concentration and the strain examined $(5,21)$.

Table 1 summarizes the results obtained with all antibiotics examined in the present study, comparing their activities when they are used alone or in combination with rifampin or oritavancin with their activities when they are used at their static concentrations (the corresponding kill curves are shown in the left panels of Fig. SP1 in the supplemental material). The addition of rifampin was clearly beneficial for every antibiotic tested, with the greatest reductions in bacterial counts observed with oxacillin, moxifloxacin, gentamicin, and oritavancin (ranked from smaller to greater effects) at $24 \mathrm{~h}$ (and also for fusidic acid but only at $5 \mathrm{~h}$ ). The addition of oritavancin was also beneficial, but the gain in activity was lower at $24 \mathrm{~h}$ than observed in combinations involving rifampin, with differences between antibiotics that were either modest or statistically nonsignificant, except for the combination oritavancin-rifampin. 


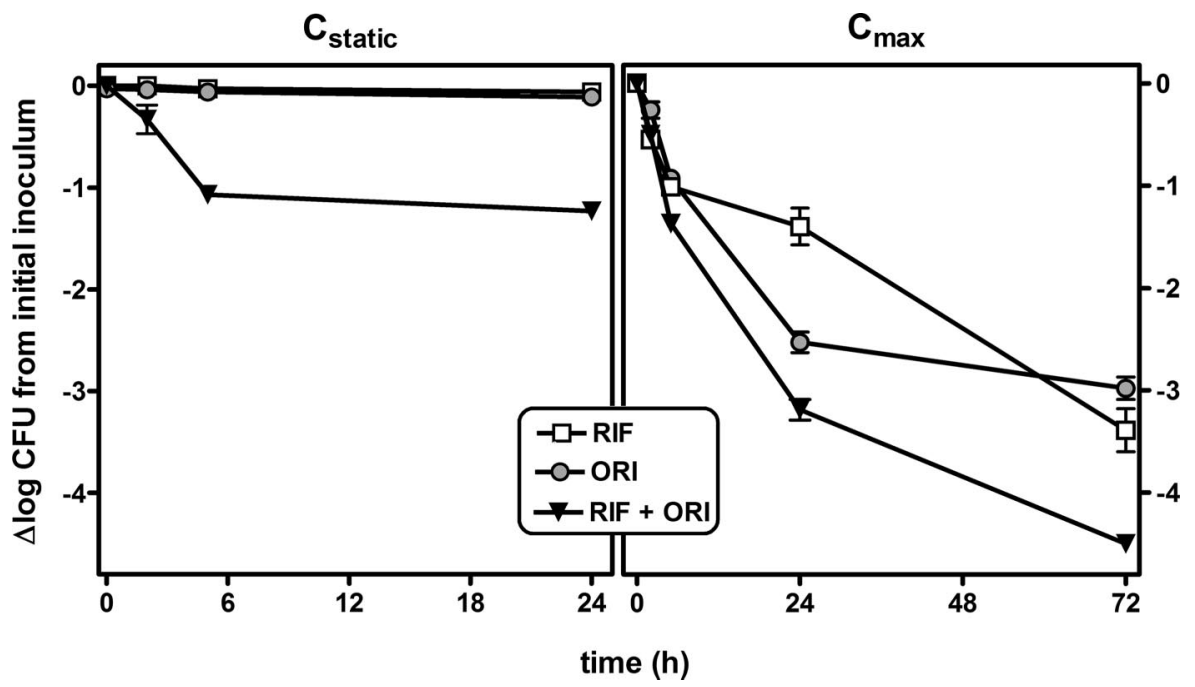

FIG. 1. Influence of time on the rate and extent of activity of rifampin (RIF), oritavancin (ORI) and of their combination (RIF+ORI) against intracellular SCVs upon incubation at a fixed extracellular concentration corresponding to the following: (i) a static effect $\left(C_{\text {static }}\right.$ as determined in the companion paper (21) (values are $0.003 \mathrm{mg} /$ liter for rifampin and $0.14 \mathrm{mg} /$ /iter for oritavancin) or (ii) their maximal concentration (total drug) observed in humans after administration of conventional doses $\left(C_{\max }\right)$ (values are $18 \mathrm{mg} /$ liter for rifampin [1] and $25 \mathrm{mg} /$ liter for oritavancin [29]) when drugs are tested alone. The ordinate is the change in the number of CFU per mg of cell protein (log scale). Values are means \pm standard deviations $(n=3)$; when not visible, the error bars are smaller than the symbols.

Table 2 shows the results obtained for antibiotics combined at concentrations mimicking the human $C_{\max }$ (total drug) and for postphagocytosis incubation times of 24 and $72 \mathrm{~h}$ (the corresponding kill curves are shown in the right panels of Fig. SP1 in the supplemental material). All combinations with rifampin led to a degree of intracellular killing similar to that obtained with rifampin at $24 \mathrm{~h}$ (1- and 1.5-log CFU decrease from the postphagocytosis inoculum) except for quinupristindalfopristin and, to a larger extent, for oritavancin, with which a greater degree of intracellular activity was obtained. Similar observations were made at $5 \mathrm{~h}$, in the sense that the reduction in bacterial counts was of the same order of magnitude (about $1 \mathrm{log}$ ) for rifampin or oritavancin alone or in combination with the other antibiotics (see Figure SP1 in supplemental material). At 72 h, slightly less activity compared to rifampin alone was observed for most drugs, but this difference reached 1 log or more for fusidic acid, clindamycin, and linezolid. Notably, the combination with oritavancin was the only one to show higher activity than rifampin alone, reaching the limit of detection $(-4.5 \operatorname{logs})$. When we then examined the combinations with oritavancin, we observed a higher activity for rifampin, gentamicin, moxifloxacin, and quinupristin-dalfopristin, with the increase in activity compared to oritavancin alone reaching for these four drugs $\sim 0.2 \log$ at $5 \mathrm{~h}$ (see Figure SP1 in the supplemental material), $\sim 0.5 \log$ at $24 \mathrm{~h}$, and at least $1 \log$ at $72 \mathrm{~h}$, respectively.

The next series of experiments aimed at further characterizing the nature of the interaction within combinations. To this effect, we used the FME method (10, 16), focusing on the combinations between rifampin, oritavancin, and moxifloxacin

TABLE 1. Comparative intracellular activities of antibiotics alone or combined with rifampin or with oritavancin against an SCV strain exposed for the indicated times at a fixed extracellular concentration giving rise to a static intracellular effect when tested alone ${ }^{a}$

\begin{tabular}{|c|c|c|c|c|c|c|c|}
\hline \multirow{3}{*}{ Drug } & \multirow{3}{*}{$\begin{array}{c}\text { Extracellular } \\
\text { concn } \\
(\mathrm{mg} / \text { liter })\end{array}$} & \multicolumn{6}{|c|}{ Intracellular activity (change in log CFU from time zero to the indicated time) ${ }^{b}$} \\
\hline & & \multicolumn{2}{|c|}{ Drug alone } & \multicolumn{2}{|c|}{ Drug with rifampin } & \multicolumn{2}{|c|}{ Drug with oritavancin } \\
\hline & & $5 \mathrm{~h}$ & $24 \mathrm{~h}$ & $5 \mathrm{~h}$ & $24 \mathrm{~h}$ & $5 \mathrm{~h}$ & $24 \mathrm{~h}$ \\
\hline Rifampin & 0.003 & $-0.03 \pm 0.01$ & $-0.06 \pm 0.02$ & NA & NA & $-1.07 \pm 0.07$ & $-1.23 \pm 0.07$ \\
\hline Vancomycin & 2.59 & $0.00 \pm 0.01$ & $0.00 \pm 0.02$ & $-0.19 \pm 0.07$ & $-0.34 \pm 0.03$ & ND & ND \\
\hline Oxacillin & 0.25 & $0.03 \pm 0.04$ & $-0.06 \pm 0.04$ & $-0.31 \pm 0.06$ & $-0.93 \pm 0.12$ & $-0.25 \pm 0.09$ & $-0.32 \pm 0.09$ \\
\hline Fusidic acid & 0.19 & $0.01 \pm 0.03$ & $0.00 \pm 0.01$ & $-0.31 \pm 0.06$ & $-0.21 \pm 0.02$ & ND & ND \\
\hline Gentamicin & 0.2 & $-0.10 \pm 0.03$ & $-0.01 \pm 0.02$ & $-0.42 \pm 0.04$ & $-0.99 \pm 0.02$ & $-0.16 \pm 0.04$ & $-0.34 \pm 0.01$ \\
\hline Clindamycin & 0.18 & $0.00 \pm 0.01$ & $-0.01 \pm 0.01$ & $-0.05 \pm 0.03$ & $-0.41 \pm 0.05$ & $-0.08 \pm 0.01$ & $-0.18 \pm 0.07$ \\
\hline Moxifloxacin & 0.23 & $-0.02 \pm 0.01$ & $-0.04 \pm 0.01$ & $-0.52 \pm 0.04$ & $-0.93 \pm 0.14$ & $-0.31 \pm 0.06$ & $-0.32 \pm 0.11$ \\
\hline Linezolid & 3.74 & $0.00 \pm 0.01$ & $-0.01 \pm 0.01$ & $-0.07 \pm 0.04$ & $-0.26 \pm 0.03$ & $-0.12 \pm 0.02$ & $-0.31 \pm 0.07$ \\
\hline Quinupristin-dalfopristin & 0.51 & $-0.01 \pm 0.01$ & $0.00 \pm 0.01$ & $-0.12 \pm 0.01$ & $-0.47 \pm 0.06$ & $-0.25 \pm 0.08$ & $-0.37 \pm 0.08$ \\
\hline Daptomycin & 0.43 & $0.01 \pm 0.02$ & $-0.01 \pm 0.02$ & $-0.18 \pm 0.11$ & $-0.32 \pm 0.13$ & $-0.16 \pm 0.07$ & $-0.36 \pm 0.04$ \\
\hline Oritavancin & 0.14 & $-0.03 \pm 0.01$ & $-0.08 \pm 0.02$ & $-1.07 \pm 0.07$ & $-1.23 \pm 0.07$ & NA & NA \\
\hline
\end{tabular}

${ }^{a}$ For the static concentration, see the companion to this paper (21).

${ }^{b}$ Values in bold highlight activities that are significantly higher than that of rifampin or oritavancin alone, as determined by analysis of variance with Dunnett's posthoc test $(P<0.05)$. NA, not applicable; ND, not done. 
TABLE 2. Comparative intracellular activities of antibiotics alone or combined with rifampin or with oritavancin against an SCV strain exposed for the indicated times at a fixed extracellular concentration corresponding to the human $C_{\max }{ }^{a}$

\begin{tabular}{|c|c|c|c|c|c|c|c|}
\hline \multirow{3}{*}{ Drug } & \multirow{3}{*}{$\begin{array}{l}\text { Extracellular } \\
\text { concn } \\
\text { (mg/liter) }\end{array}$} & \multicolumn{6}{|c|}{ Intracellular activity (change in log CFU from time zero to the indicated time) ${ }^{b}$} \\
\hline & & \multicolumn{2}{|c|}{ Drug alone } & \multicolumn{2}{|c|}{ Drug with rifampin } & \multicolumn{2}{|c|}{ Drug with oritavancin } \\
\hline & & $24 \mathrm{~h}$ & $72 \mathrm{~h}$ & $24 \mathrm{~h}$ & $72 \mathrm{~h}$ & $24 \mathrm{~h}$ & $72 \mathrm{~h}$ \\
\hline Rifampin & 18 & $-1.40 \pm 0.18$ & $-3.39 \pm 0.21$ & NA & NA & $-3.19 \pm 0.10$ & -4.50 \\
\hline Vancomycin & 50 & $-0.11 \pm 0.04$ & $-1.35 \pm 0.08$ & $-1.42 \pm 0.03$ & $-2.75 \pm 0.14$ & ND & ND \\
\hline Oxacillin & 64 & $-0.06 \pm 0.04$ & $-1.31 \pm 0.06$ & $-1.28 \pm 0.05$ & $-2.81 \pm 0.01$ & $-2.61 \pm 0.11$ & $-3.25 \pm 0.06$ \\
\hline Fusidic acid & 30 & $-0.33 \pm 0.07$ & $-0.87 \pm 0.04$ & $-1.06 \pm 0.01$ & $-2.44 \pm 0.01$ & ND & ND \\
\hline Gentamicin & 18 & $-0.44 \pm 0.09$ & $-1.54 \pm 0.09$ & $-1.35 \pm 0.06$ & $-2.92 \pm 0.01$ & $-2.90 \pm 0.09$ & $-3.70 \pm 0.13$ \\
\hline Clindamycin & 4 & $-0.27 \pm 0.10$ & $-1.30 \pm 0.08$ & $-1.31 \pm 0.03$ & $-2.36 \pm 0.10$ & $-2.63 \pm 0.12$ & $-3.54 \pm 0.11$ \\
\hline Moxifloxacin & 4 & $-1.20 \pm 0.05$ & $-2.53 \pm 0.04$ & $-1.22 \pm 0.06$ & $-2.94 \pm 0.07$ & $-3.26 \pm 0.13$ & $-4.18 \pm 0.16$ \\
\hline Linezolid & 16 & $-0.39 \pm 0.01$ & $-1.52 \pm 0.08$ & $-1.05 \pm 0.07$ & $-1.86 \pm 0.11$ & $-2.73 \pm 0.09$ & $-3.60 \pm 0.06$ \\
\hline Quinupristin-dalfopristin & 11 & $-0.22 \pm 0.03$ & $-2.06 \pm 0.16$ & $-1.80 \pm 0.05$ & $-2.63 \pm 0.04$ & $-2.82 \pm 0.15$ & $-4.18 \pm 0.32$ \\
\hline Daptomycin & 57 & $-0.39 \pm 0.02$ & $-1.38 \pm 0.01$ & $-1.50 \pm 0.06$ & $-2.92 \pm 0.06$ & $-2.59 \pm 0.05$ & $-2.97 \pm 0.16$ \\
\hline Oritavancin & 25 & $-2.53 \pm 0.10$ & $-2.98 \pm 0.11$ & $-3.19 \pm 0.10$ & -4.50 & NA & NA \\
\hline
\end{tabular}

${ }^{a}$ Human $C_{\max }$ corresponds to total drug. See Table 1 in the companion paper for references (21).

${ }^{b}$ Values in bold highlight activities that are significantly higher, and values in italics highlight those that are significantly lower than the activity of rifampin or oritavancin alone, as determined by analysis of variance with Dunnett's posthoc test $(P<0.05)$. NA, not applicable; ND, not done.

since these drugs proved very active in our model when used alone, with clear indications of a favorable effect of their combinations when tested at fixed concentrations (see above). Maximal effects used for calculating concentrations were a 1.72-log and 1.32-log CFU decrease for rifampin and moxifloxacin, respectively (21). As studies with oritavancin alone revealed a bimodal dose-effect relationship (21), we considered for this case two $E_{\max }$ values (at 0.43 - and 3.13-log reduction), corresponding to the two successive plateaus observed in these experiments. We first validated the approach by examining to what extent the observed values of FME (FME $\left.{ }_{\text {obs }}\right)$ were linearly correlated with the concentration of these antibiotics when tested alone. This is shown in Fig. 2 with an overall correlation coefficient $\left(R^{2}\right)$ of 0.80 for all four conditions (oritavancin using $E_{\max }$ values corresponding to low and high concentrations; moxifloxacin; and rifampin) and a slope of $1.10 \pm 0.13$ between the observed and expected FME values. The antibiotics were then combined two by two, and the resulting FME values of the combinations are shown in Fig. 3. The combination of rifampin with oritavancin was synergistic at all concentrations tested when examining the range of oritavancin concentrations corresponding to the first plateau of activity when tested alone (up to about $5 \mathrm{mg} / \mathrm{liter}$ ). This combination maintained a synergistic effect at higher oritavancin concentrations (in the range of 8 to $45 \mathrm{mg} / \mathrm{liter}$, with rifampin varying from 0.04 to $0.007 \mathrm{mg} /$ liter) but became additive above and below these values. The combination of moxifloxacin with oritavancin was indifferent for the lowest oritavancin concentrations investigated (FME of $<1$ but above the curve of the drugs alone) but became additive (FME close to 1) at higher concentrations, with a synergistic effect (FME of $>1$ ) when moxifloxacin and oritavancin were used at a 0.3/19 $\mathrm{mg} / \mathrm{liter}$ concentration ratio. Combining moxifloxacin with rifampin showed essentially an additive effect (FME was close to 1 over the whole range of concentrations ratios investigated).

\section{DISCUSSION}

The present study contributes to ongoing systematic efforts at defining conditions that are likely to improve the therapeutic outcome of intracellular infections due to SCV strains by means of antibiotic combinations $(2,3)$. The model used in this work is derived directly from the model that we used previously to establish key pharmacological properties of antistaphylococcal antibiotics against intracellular $S$. aureus with normal-phenotype and SCV strains, using a well-controlled environment and minimizing interferences by host-related factors $(5,21)$.

The first key observation made here is that the combination of either rifampin or oritavancin with a series of commonly

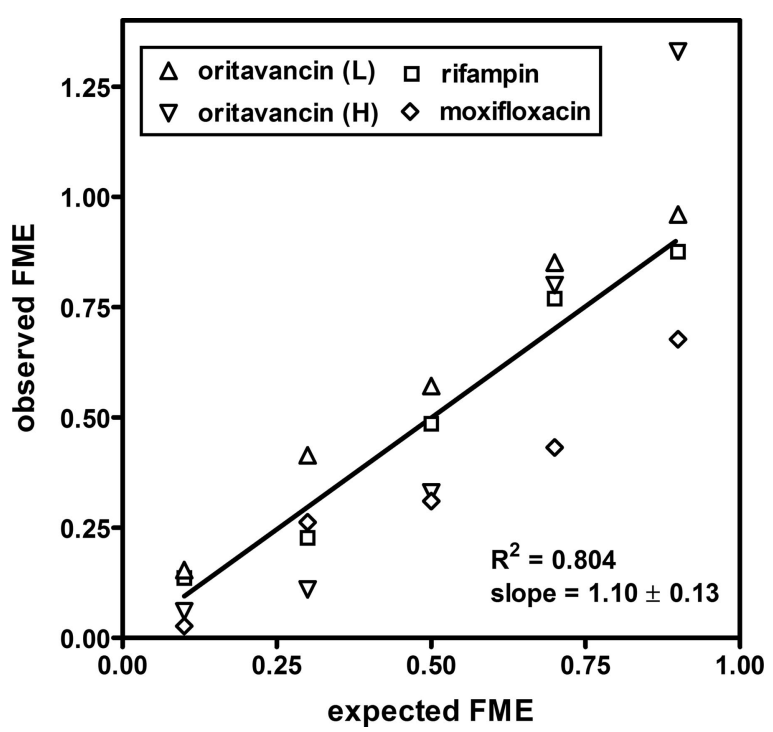

FIG. 2. Correlation between observed and expected FMEs of four antibiotics toward intracellular SCVs when used alone. Antibiotics were added at concentrations calculated to yield FME values of 0.1 , $0.3,0.5,0.7$, and 0.9 , and the actual FME calculated based on the observed activities at $24 \mathrm{~h}$ was compared to the maximal activities as determined in dose-dependence experiments $\left[E_{\max }\right.$ values of $-1.72 \mathrm{log}$ CFU for rifampin and $-1.32 \log$ CFU for moxifloxacin; for oritavancin, two successive $E_{\max }$ values of $-0.43 \log$ CFU at low (L) and -3.13 $\log \mathrm{CFU}$ at high $(\mathrm{H})$ concentrations were considered based on the observation of a bimodal concentration-effect relationship (see Table 2 in reference 21)]. 
A
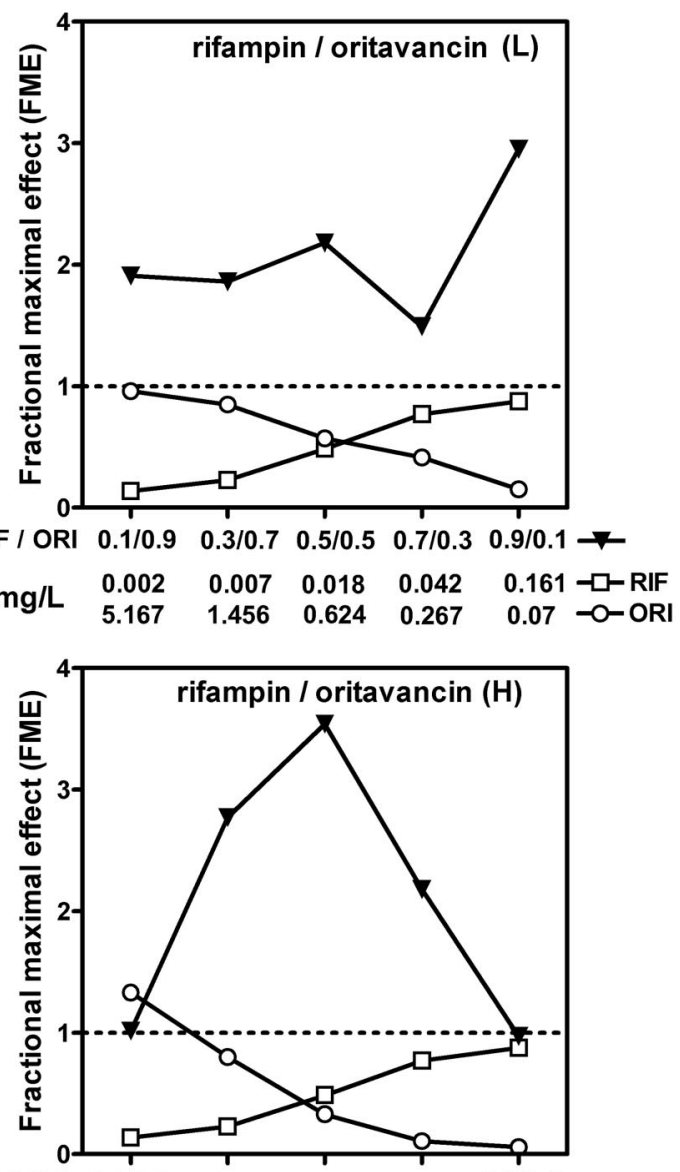

RIF / ORI $\quad 0.1 / 0.9 \quad 0.3 / 0.7 \quad 0.5 / 0.5 \quad 0.7 / 0.3 \quad 0.9 / 0.1$

$\begin{array}{lllllllll}\mathrm{mg} / \mathrm{L} & 0.002 & 0.007 & 0.018 & 0.042 & 0.161 & -\square-\mathrm{RIF}\end{array}$

$\mathrm{mg} / \mathrm{L} \quad \begin{array}{llllll}171.72 & 44.52 & 19.08 & 8.17 & 2.12 & -0-O R I\end{array}$

B
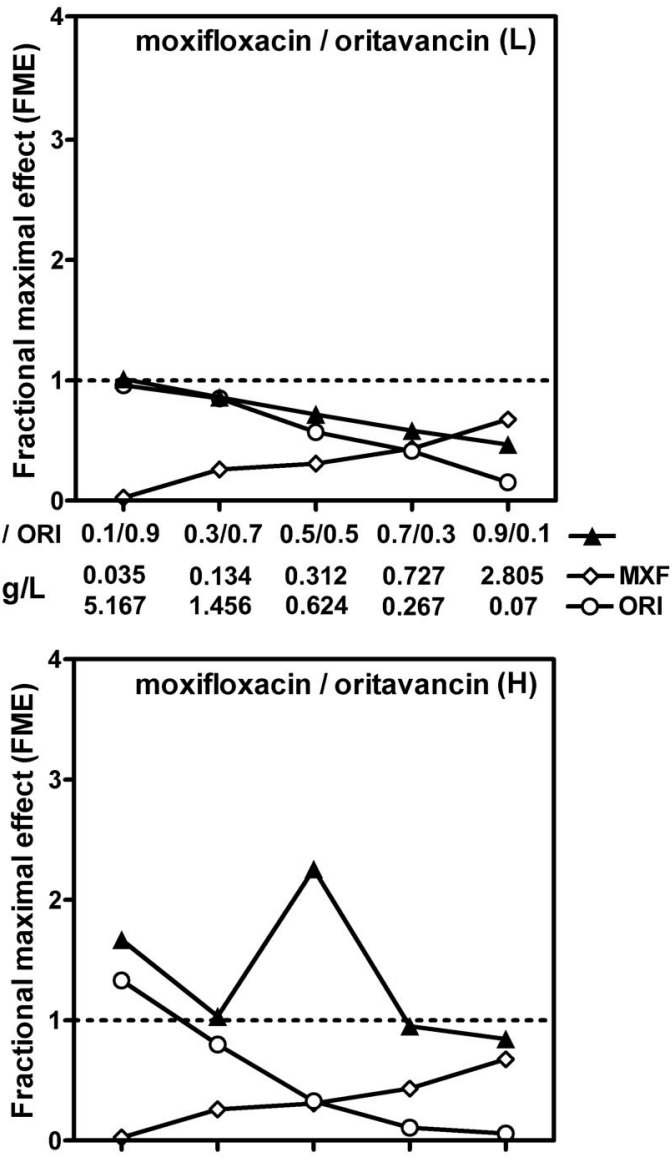

$\begin{array}{llllllll}M X F / O R I & 0.1 / 0.9 & 0.3 / 0.7 & 0.5 / 0.5 & 0.7 / 0.3 & 0.9 / 0.1 & \text { ד }\end{array}$

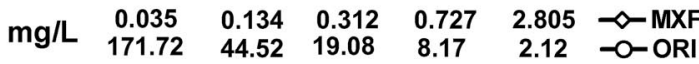

C

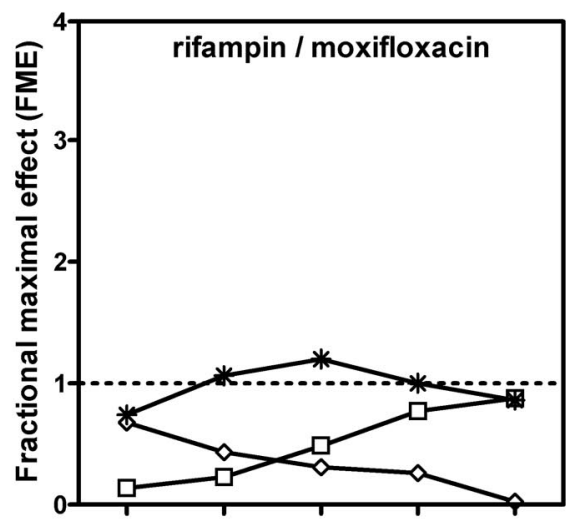

$\begin{array}{ccccccc}\text { RIF / MXF } & 0.1 / 0.9 & 0.3 / 0.7 & 0.5 / 0.5 & 0.7 / 0.3 & 0.9 / 0.1 & * \\ \text { mg/L } & 0.002 & 0.007 & 0.018 & 0.042 & 0.161 & \text { - } \\ 2.805 & 0.727 & 0.312 & 0.134 & 0.035 & \text { RIF } \\ \text { MXF }\end{array}$

FIG. 3. FME plots of antibiotics against intracellular SCV. In each graph, the abscissa shows the concentrations of antibiotics tested, indicated above the graphs, either alone or in combination and expressed as follows: (i) the five expected FME values examined for antibiotics alone (0.1 to 0.9 or 0.9 to 0.1 ) and the ratios of the expected FME values contributed by each antibiotic (from $0.1 / 0.9$ to $0.9 / 0.1$ ) for antibiotic combinations; and (ii) the corresponding concentrations (in $\mathrm{mg} /$ liter) used. The ordinate shows the value of the observed FME for each antibiotic alone (open symbols) or for the combination [ $\mathrm{FME}_{\mathrm{obs}(A+B)}$; shown as closed symbols in $\mathrm{A}$ and $\mathrm{B}$ and as stars in $\left.\mathrm{C}\right]$. Values of $\mathrm{FME}_{\mathrm{obs}(A+B)}$ that are $>1$ denote a synergistic effect; values equal to 1 indicate an additive effect; values of $<1$ but higher than $\mathrm{FME}$ of the antibiotics alone $\left(\mathrm{FME}_{\mathrm{obs}} \mathrm{A}\right.$ or $\left.\mathrm{FME}_{\mathrm{obs} B}\right)$ indicate an indifferent effect (a value of $<1$ and below $\mathrm{FME}_{\mathrm{obs} A}$ or $\mathrm{FME}_{\mathrm{obs} B}$ would indicate an antagonistic effect, which was not observed here) (10). For oritavancin, two ranges of concentrations were examined based on the observation of a bimodal concentration-effect relationship with two successive $E_{\max }$ values of $-0.43 \log$ CFU at low (L) and $-3.13 \log$ CFU at high (H) concentrations (see Table 2 in reference 21 ). 
used antistaphylococcal agents may produce largely divergent effects, based on the following observations: (i) globally favorable effects were observed with rifampin at low concentrations only (and its activity was partly diminished when combined with the same antibiotics at high concentrations); and (ii) oritavancin produced favorable effects at both low and high concentrations. A survey of the adjunctive use of rifampin for the treatment of $S$. aureus infections suggests antagonism or indifference with $\beta$-lactams, fluoroquinolones, linezolid, or daptomycin; positive or negative effects, depending on the model, for vancomycin, fusidic acid, or clindamycin; and synergy only for quinupristin-dalfopristin (24). These conclusions are largely supported in our model as well, except that we did not observe a marked advantage when we combined rifampin with quinupristin-dalfopristin. Our results also show that the concentrations at which these effects are measured were critical and that favorable effects with rifampin may only clearly appear when the drug is used at suboptimal concentrations. Our observation that the combination of oritavancin with other antistaphylococcal drugs is systematically favorable is in line with observations made with $S$. aureus strains with various resistance phenotypes when they are exposed to combinations of oritavancin with gentamicin, vancomycin, or rifampin $(6,7,18)$.

A second key observation is that the combination of rifampin with oritavancin was markedly and almost always synergistic, with a global effect suggesting that near eradication of an intracellular SCV strain could be obtained. If confirmed in other models, this observation could have considerable value for the following reasons: (i) persistence of SCVs is considered as one of the significant causes of persistence and relapses in staphylococcal infections (31); (ii) these variants could often be intracellular in vivo (30) and appear only poorly susceptible to antibiotics when used alone in our model (21). One caveat of our observations, however, is that eradication might require extracellular concentrations that may not be attainable in vivo under clinically acceptable conditions. It is nevertheless pertinent that a large decrease in the intracellular inoculum was observed at lower, more physiologically relevant concentrations of rifampin and oritavancin.

The model used in the present study, however, has some features that may limit its significance. As analyzed in our previous publications $(5,14,15)$, these limitations include a lack of dynamic aspect (i.e., the antibiotics are maintained at fixed concentrations throughout the observation period), which could be important here in view of the duration of the experiments, as well as the impossibility of assessing the importance of protein binding, a point that will need careful attention for oritavancin since this antibiotic is highly protein bound $(25,32)$. Our study is also limited to a single strain, preventing us from drawing general conclusions on the extent of synergy reached, which may depend on the strain, its susceptibility to antibiotics, its intracellular growth, or the metabolic defect responsible for its SCV character, as illustrated by comparing the data generated here with those obtained for two hemin-dependent SCV strains with higher levels of intracellular growth (2).

Findings from our study provide further impetus to investigate the mechanisms that result in synergistic activity against intracellular bacteria. We may, however, speculate that the systematically favorable effects seen with oritavancin could be related (i) to its intense bactericidal effect, probably due to membrane-destabilizing properties $(11,17)$ that may favor access to target for other antibiotics (6), and/or (ii) to the high concentration that oritavancin reaches in the phagolysosomes (28) where SCV strains also locate (21). The negative effect exerted by all antistaphylococcal drugs, except oritavancin, toward rifampin when tested at high concentrations also requires further explanation. Unfortunately, we could not obtain direct information about drug stability and the absence of drug-drug interactions within macrophages during the prolonged exposure times needed to assess antibiotic activity against intracellular SCVs. However, the fact that rifampin is stable for several days at $37^{\circ} \mathrm{C}$ in a susceptibility testing medium (13), the lack of specificity with respect to the pharmacochemical class of the drugs tested, and the enhancing effect of oritavancin on the activity of several of these antibiotics make interpretations based on chemical alteration or physical inactivation of the drugs unlikely.

In spite of these uncertainties, and pending additional data gathered from more pertinent models, our data highlight the interest of combining antibiotics having an appropriate pharmacokinetic/pharmacodynamic profile against $S$. aureus $(5,27)$ and showing activity against nongrowing bacteria $(19,20)$ to successfully control and reduce intracellular infection by SCVs. This study may trigger further evaluation of antibiotic combinations against intracellular bacteria and ultimately may help in the design of pertinent clinical trials.

\section{ACKNOWLEDGMENTS}

This work was supported by the Institut d'encouragement de la Recherche Scientifique et de l'Innovation de Bruxelles (IRSIB)/Instituut ter Bevordering van het Wetenschappelijk Onderzoek en de Innovatie van Brussel (IWOIB) of the Brussels Capital Region, within the framework of the Research in Brussels program, by the Fonds de la Recherche Scientifique Médicale (grants 3.4.597.06 and 3.4.639.09), and by a grant-in-aid from Targanta Therapeutics Corp. H.A.N. was successively a postdoctoral fellow of the IRSIB/IWOIB and of the Fonds de la Recherche Scientifique-Fonds National de la Recherche Scientifique (FRS-FNRS); F.V.B. is Maître de Recherches of the Belgian FRS-FNRS.

We are grateful to J. J. Schentag (University of Buffalo, Buffalo, NY) for valuable advice in the design of our experiments and useful discussions. M. C. Cambier and C. Misson provided dedicated technical assistance.

\section{REFERENCES}

1. Amsden, G. W. 2000. Tables of antimicrobial agents pharmacology, p. 635700. In G. L. Mandell, J. E. Bennett, and R. Dolin (ed.), Principles and practice of infectious diseases. Churchill Livingstone, New York, NY.

2. Baltch, A. L., W. J. Ritz, L. H. Bopp, P. Michelsen, and R. P. Smith. 2008. Activities of daptomycin and comparative antimicrobials, singly and in combination, against extracellular and intracellular Staphylococcus aureus and its stable small-colony variant in human monocyte-derived macrophages and in broth. Antimicrob. Agents Chemother. 52:1829-1833.

3. Baltch, A. L., W. J. Ritz, L. H. Bopp, P. B. Michelsen, and R. P. Smith. 2007. Antimicrobial activities of daptomycin, vancomycin, and oxacillin in human monocytes and of daptomycin in combination with gentamicin and/or rifampin in human monocytes and in broth against Staphylococcus aureus. Antimicrob. Agents Chemother. 51:1559-1562.

4. Bamberger, D. M., B. L. Herndon, M. Dew, R. P. Chern, H. Mitchell, L. E. Summers, R. F. Marcus, S. C. Kim, and P. R. Suvarna. 1997. Efficacies of ofloxacin, rifampin, and clindamycin in treatment of Staphylococcus aureus abscesses and correlation with results of an in vitro assay of intracellular bacterial killing. Antimicrob. Agents Chemother. 41:1178-1181.

5. Barcia-Macay, M., C. Seral, M. P. Mingeot-Leclercq, P. M. Tulkens, and F. Van Bambeke. 2006. Pharmacodynamic evaluation of the intracellular activities of antibiotics against Staphylococcus aureus in a model of THP-1 macrophages. Antimicrob. Agents Chemother. 50:841-851. 
6. Belley, A., E. Neesham-Grenon, F. F. Arhin, G. A. McKay, T. R. Parr, Jr., and G. Moeck. 2008. Assessment of the synergistic effects of oritavancin tested in combination with other antimicrobial agents against Staphylococcus aureus by time-kill methodology. Antimicrob. Agents Chemother. 52:3820 3822 .

7. Belley, A., E. Neesham-Grenon, S. Beaulieu, G. McKay, F. Arhin, T. R. Parr, Jr., and G. Moeck. 2007. Synergistic effects of oritavancin tested in combination with other agents, abstr. E-1619. Abstr. 48th Intersci. Conf. Antimicrob. Agents Chemother. American Society for Microbiology, Washington, DC.

8. Besier, S., J. Zander, B. C. Kahl, P. Kraiczy, V. Brade, and T. A. Wichelhaus. 2008. The thymidine-dependent small-colony-variant phenotype is associated with hypermutability and antibiotic resistance in clinical Staphylococcus aureus isolates. Antimicrob. Agents Chemother. 52:2183-2189.

9. Crandon, J., and D. P. Nicolau. 2008. Oritavancin: a potential weapon in the battle against serious gram-positive pathogens. Future Microbiol. 3:251-263.

10. Desbiolles, N., L. Piroth, C. Lequeu, C. Neuwirth, H. Portier, and P. Chavanet. 2001. Fractional maximal effect method for in vitro synergy between amoxicillin and ceftriaxone and between vancomycin and ceftriaxone agains Enterococcus faecalis and penicillin-resistant Streptococcus pneumoniae. Antimicrob. Agents Chemother. 45:3328-3333.

11. Domenech, O., F. Van Bambeke, P. M. Tulkens, and M. P. MingeotLeclercq. 2008. Comparative study of membrane permabilization induced by oritavancin versus vancomycin in liposomes: importance of the acidic character of the membrane, abstr.C1-199. Abstr. 48th Intersci. Confer. Antimicrob. Agents Chemother. (ICAAC)-Infect. Dis. Soc. Am. (IDSA) 46th Annu. Meet. American Society for Microbiology and Infectious Diseases Society of America, Washington, DC.

12. Eliopoulos, G. M., and C. T. Eliopoulos. 1988. Antibiotic combinations: should they be tested? Clin. Microbiol. Rev. 1:139-156.

13. Griffith, M. E., and H. L. Bodily. 1992. Stability of antimycobacterial drugs in susceptibility testing. Antimicrob. Agents Chemother. 36:2398-2402.

14. Lemaire, S., A. Olivier, F. Van Bambeke, P. M. Tulkens, P. C. Appelbaum, and Y. Glupczynski. 2008. Restoration of susceptibility of intracellular methicillin-resistant Staphylococcus aureus to $\beta$-lactams: comparison of strains, cells, and antibiotics. Antimicrob. Agents Chemother. 52:2797-2805.

15. Lemaire, S., F. Van Bambeke, M. P. Mingeot-Leclercq, and P. M. Tulkens. 2005. Activity of three $\beta$-lactams (ertapenem, meropenem and ampicillin) against intraphagocytic Listeria monocytogenes and Staphylococcus aureus. J. Antimicrob. Chemother. 55:897-904

16. Li, R. C., J. J. Schentag, and D. E. Nix. 1993. The fractional maximal effect method: a new way to characterize the effect of antibiotic combinations and other nonlinear pharmacodynamic interactions. Antimicrob. Agents Chemother. 37:523-531.

17. McKay, G. A., I. Fadhil, S. Beaulieu, S. Ciblat, A. R. Far, G. Moeck, and T. R. Parr, Jr. 2006. Oritavancin disrupts transmembrane potential and membrane integrity concomitantly with cell killing in Staphylococcus aureus and vancomycin-resistant enterococci, abstr. C1-682. Abstr. 46th Intersci. Conf. Antimicrob. Agents Chemother. American Society for Microbiology, Washington, DC.

18. Mercier, R. C., H. H. Houlihan, and M. J. Rybak. 1997. Pharmacodynamic evaluation of a new glycopeptide, LY333328, and in vitro activity against
Staphylococcus aureus and Enterococcus faecium. Antimicrob. Agents Chemother. 41:1307-1312.

19. Mercier, R. C., C. Stumpo, and M. J. Rybak. 2002. Effect of growth phase and $\mathrm{pH}$ on the in vitro activity of a new glycopeptide, oritavancin (LY333328), against Staphylococcus aureus and Enterococcus faecium. J. Antimicrob. Chemother. 50:19-24.

20. Murillo, O., A. Domenech, A. Garcia, F. Tubau, C. Cabellos, F. Gudiol, and J. Ariza. 2006. Efficacy of high doses of levofloxacin in experimental foreignbody infection by methicillin-susceptible Staphylococcus aureus. Antimicrob. Agents Chemother. 50:4011-4017.

21. Nguyen, H. A., O. Denis, A. Vergison, A. Theunis, P. M. Tulkens, M. J. Struelens, and F. Van Bambeke. 2009. Intracellular activity of antibiotics in a model of human THP-1 macrophages infected by a Staphylococcus aureus small-colony variant strain isolated from a cystic fibrosis patient: pharmacodynamic evaluation and comparison with isogenic normal-phenotype and revertant strains. Antimicrob. Agents Chemother. 53:1434-1442.

22. Patel, R., M. S. Rouse, K. E. Piper, F. R. Cockerill III, and J. M. Steckelberg. 1998. In vitro activity of LY333328 against vancomycin-resistant enterococci, methicillin-resistant Staphylococcus aureus, and penicillin-resistant Streptococcus pneumoniae. Diagn. Microbiol. Infect. Dis. 30:89-92.

23. Patel, S. M., and L. D. Saravolatz. 2006. Monotherapy versus combination therapy. Med. Clin. N. Am. 90:1183-1195.

24. Perlroth, J., M. Kuo, J. Tan, A. S. Bayer, and L. G. Miller. 2008. Adjunctive use of rifampin for the treatment of Staphylococcus aureus infections: a systematic review of the literature. Arch. Intern. Med. 168:805-819.

25. Rowe, P. A., and T. J. Brown. 2001. Protein binding of 14C-oritavancin. Abstr. 41th Intersci. Conf. Antimicrob. Agents Chemother., abstr. A-2193.

26. Schwalbe, R. S., A. C. McIntosh, S. Qaiyumi, J. A. Johnson, R. J. Johnson, K. M. Furness, W. J. Holloway, and L. Steele-Moore. 1996. In vitro activity of LY333328, an investigational glycopeptide antibiotic, against enterococci and staphylococci. Antimicrob. Agents Chemother. 40:2416-2419.

27. Van Bambeke, F., M. Barcia-Macay, S. Lemaire, and P. M. Tulkens. 2006 Cellular pharmacodynamics and pharmacokinetics of antibiotics: current views and perspectives. Curr. Opin. Drug Discov. Devel. 9:218-230.

28. Van Bambeke, F., S. Carryn, C. Seral, H. Chanteux, D. Tyteca, M. P. Mingeot-Leclercq, and P. M. Tulkens. 2004. Cellular pharmacokinetics and pharmacodynamics of the glycopeptide antibiotic oritavancin (LY333328) in a model of J774 mouse macrophages. Antimicrob. Agents Chemother. 48: 2853-2860.

29. Van Bambeke, F., Y. Van Laethem, P. Courvalin, and P. M. Tulkens. 2004. Glycopeptide antibiotics: from conventional molecules to new derivatives. Drugs 64:913-936.

30. von Eiff, C. 2008. Staphylococcus aureus small colony variants: a challenge to microbiologists and clinicians. Int. J. Antimicrob. Agents 31:507-510.

31. von Eiff, C., G. Peters, and K. Becker. 2006. The small colony variant (SCV) concept: the role of staphylococcal SCVs in persistent infections. Injury 37(Suppl. 2):S26-S33.

32. Zhanel, G. G., I. D. Kirkpatrick, D. J. Hoban, A. M. Kabani, and J. A. Karlowsky. 1998. Influence of human serum on pharmacodynamic properties of an investigational glycopeptide, LY333328, and comparator agents against Staphylococcus aureus. Antimicrob. Agents Chemother. 42:24272430. 